\title{
Astromesitius, a new genus of Mesitiinae (Hymenoptera, Bethylidae) from the Old World
}

\author{
Diego N. BARBOSA ${ }^{1, *} \&$ Celso O. AZEVEDO ${ }^{2}$ \\ 1,2 Universidade Federal do Espírito Santo, Departamento de Ciências Biológicas, \\ Av. Fernando Ferrari 514, Goiabeiras, 29.075-910, Vitória, ES, Brazil. \\ *Corresponding author: mesitiinae@gmail.com \\ ${ }^{2}$ Email: bethylidae@gmail.com \\ ${ }^{1}$ urn:lsid:zoobank.org:author:95D76114-BF6C-4715-881B-10F7B2ACD2D0 \\ ${ }^{2}$ urn:1sid:zoobank.org:author:CD98B489-7611-4A33-AF53-3016B9DA1617
}

\begin{abstract}
Literature about Mesitiinae Kieffer, 1914 has not been treated extensively from a taxonomic viewpoint in comparison with other subfamilies in Bethylidae Latreille, 1802. Our research on species of Metrionotus Móczár, 1970, Clytrovorus Nagy, 1972 and Sulcomesitius Móczár, 1970 revealed a new hypopygium shape pattern, namely a 'star-shaped' hypopygium, which is characteristic of a new genus, Astromesitius gen. nov., with two new species Astromesitius thionyi gen. et sp. nov. and Astromesitius olavoi gen. et sp. nov. The descriptions of both new species are based on male specimens collected in Thailand and the United Arab Emirates. Astromesitius quatei (Móczár, 1977) gen. et comb. nov. is designated as type species for the new genus, which is erected for a total of seven species. The main diagnostic characteristics are the head longer than wide; a clypeus with a median lobe quadrate; an antenna with distinct long setae, with pedicel caliciform, and with flagellomeres long and caliciform; pronotum and anteromesoscutum with longitudinal sulcus indistinct or absent; metapectal-propodeal complex with posterior projection hardly distinct or absent; hypopygium star-shaped; genitalia with aedeagus slender and fusiform.
\end{abstract}

Keywords. New genus, hypopygium, 'star-shaped', new species, United Arab Emirates.

Barbosa D.N. \& Azevedo C.O. 2019 Astromesitius, a new genus of Mesitiinae (Hymenoptera, Bethylidae) from the Old World. European Journal of Taxonomy 587: 1-20. https://doi.org/10.5852/ejt.2019.587

\section{Introduction}

Mesitiinae Kieffer, 1914 currently contains 175 valid species in 17 genera (Azevedo et al. 2018). While revising the 154 types of the 240 names available in this subfamily, an unreported star-shaped hypopygium was discovered in a few species of Sulcomesitius Móczár, 1970, Clytrovorus Nagy, 1972 and Metrionotus Móczár, 1970. This kind of hypopygium was not described by Argaman (2003) in his synopsis of Mesitiinae, within which he defined the hypopygium as the most important structure to establish the genera. For this reason, the present paper proposes a new genus for the species of Mesitiinae with star-shaped hypopygium. 


\section{Material and methods}

\section{Repositories}

The names of collection managers are given in brackets.

BPBM = Bernice Pauahi Bishop Museum, USA (James Boome)

QBG = Queen Sirikit Botanical Garden, Thailand (Wichai Srisuka)

UFES = Universidade Federal do Espírito Santo, Brazil (Marcelo Tavares)

Thai specimens were collected within the scope of the project 'Thailand Inventory Group for Entomological Research' (grant \#DEB-0542864) coordinated by Michael Sharkey from the University of Kentucky (USA), and Arabian specimens within the project 'United Arab Emirates Insect Project' coordinated by Antonius van Harten.

The terms applied to the body structures follow Azevedo et al. (2018); those related to the integument follow Harris (1979).

\section{Abbreviations}

$\mathrm{HE}=$ length of the eye in lateral view

$\mathrm{LFW}=$ length of the forewing

$\mathrm{LH}=$ length of the head including clypeus in dorsal view

$\mathrm{VOL}=$ vertex-ocular line in dorsal view

The descriptions and key were elaborated with DELTA (Descriptive Language for Taxonomy) according to Dallwitz et al. (1993). The genera of Mesitiinae were identified using the key proposed by Azevedo et al. (2018).

The illustrations were obtained using Leica MZ80 stereo microscope. Images were obtained using Leica MD2500 microscope magnifying glass attached to a Leica DFC 495 video camera; the program Leica LAS (Leica Application Suite ver. 3.6.0; Microsystems by Leica (Switzerland) Limited) was used to capture individual images under the Scalable and Modular Dome Illumination System by Kawada \& Buffington (2016). Images were combined using Helicon Focus software (ver. 4.2.9); render method was based on Method C (Pyramid). All illustrations and plates were produced in software for image editing and vectorization using adjustments (e.g., levels, shadows / highlights), tools (e.g., healing brush, clone stamp) and filters (e.g., unsharp mask).

\section{Results}

Class Hexapoda Blainville, 1816

Order Hymenoptera Linnaeus, 1758

Superfamily Chrysidoidea Linnaeus, 1761

Family Bethylidae Latreille, 1802

Subfamily Mesitiinae Kieffer, 1914

Astromesitius gen. nov. urn:lsid:zoobank.org:act:AC77A4BF-FC3A-481F-BD18-8175DC3CEE5B

Figs $1-7$

\section{Type species}

Sulcomesitius quatei Móczár, 1977. 


\section{Etymology}

The name Astromesitius is composed from the Greek (and Latin) 'aster', which means 'star', and Mesitius, the type genus of Mesitiinae. The name refers to the star-shaped hypopygium of the new genus.

\section{Description}

\section{Male}

Body sparsely foveolate (Fig. 1E). Head longer than wide, malar space convergent anteriorly and as long as VOL. Clypeus with median lobe quadrate. Antenna with distinct sparse setae, pedicel caliciform, flagellomeres longer than wide and caliciform. Eye setose, circular and small, HE usually about one third of LH. Ocelli small. Dorsal pronotal area with longitudinal sulcus hardly distinct or absent. Anteromesoscutum without longitudinal sulcus. Metapectal-propodeal complex with posterior projection hardly distinct or absent. Macropterous. Hypopygium with posterior margin with short and acute branches, with lateral corner projecting and well defined, general shape resembling a star. Genitalia with ventral arm of paramere longer and wider than dorsal arm, aedeagus slender, fusiform.

\section{Female}

Not available for description.

\section{Remarks}

The main characteristic to recognize Astromesitius gen. nov. is the hypopygium shape. The star-shaped hypopygium is a unique shape within Mesitiinae. Additionally, the sparsely foveolate integument surface, the sparse pubescence of the antenna, which is of medium length to long, and the hardly distinct or absent longitudinal pronotal sulcus are also important in recognizing the genus. These characteristics are further discussed in the Discussion.

\section{Distribution}

Afrotropical: United Arab Emirates and South Africa. Oriental: Thailand and Vietnam. Palaearctic: Czech Republic, Hungary, Italy, Romania, Russia and Slovakia.

\section{Included species}

Astromesitius carbonarius (Móczár, 1970) gen. et comb. nov. from Metrionotus Astromesitius indistintus (Barbosa \& Azevedo, 2011) gen. et comb. nov. from Metrionotus Astromesitius minutissimus (Móczár, 1971) gen. et comb. nov. from Metrionotus Astromesitius mutilloides (Costa, 1864) gen. et comb. nov. from Clytrovorus Astromesitius olavoi gen. et sp. nov. Astromesitius quatei (Móczár, 1977) gen. et comb. nov. from Sulcomesitius Astromesitius thionyi gen. et sp. nov.

\section{Key to the males of Astromesitius gen. nov.}

1. Metapectal-propodeal complex projection absent (Fig. 1B-C, E); posterior hypopygeal branches slender (Fig. 2B, D)

- Metapectal-propodeal complex projection present (Fig. 1A, D, F); posterior hypopygeal branches wide (Fig. 2A, C, E)

2. Longitudinal ridge between metapostnotal median carina and metapostnotal-propodeal carina present (Fig. 1C) A. carbonarius (Móczár, 1970) gen. et comb. nov.

- Longitudinal ridge between metapostnotal median carina and metapostnotal-propodeal carina absent (Fig. 1B, E) 
3. Metapostnotal surface polished; metapostnotal median carina incomplete; propodeal declivity coriaceous and without lateral carina (Fig. 1B) A. thionyi gen. et sp. nov.

- Metapostnotal surface areolate; metapostnotal median carina complete; propodeal declivity areolate and with lateral carina (Fig. 1E) A. minutissimus (Móczár, 1971) gen. et comb. nov.

4. Body surface distinctly foveolate; dorsal pronotal area with longitudinal sulcus (Fig. 1F) 5

- Body surface distinctly foveolate; dorsal pronotal area without longitudinal sulcus (Fig. 1A, D) ....

5. Hypopygium longer than wide (Fig. 2E); cuspis ventral arm wide; aedeagus without outer distal projection (Fig. 3F)

A. quatei (Móczár, 1977) gen. et comb. nov.

- Hypopygium as long as wide; cuspis arms slender; aedeagus with outer distal projection A. mutilloides (Costa, 1864) gen. et comb. nov.

6. Head and mesosoma dark castaneous nearly black (Fig. 4A); lateral hypopygeal projection long (Fig. 2A); aedeagus with outer projection at apex (Fig. 3A)

A. olavoi gen. et sp. nov.

- Head and mesosoma orange (Fig. 4D); lateral hypopygeal projection short (Fig. 2C); aedeagus without outer projection at apex (Fig. 3D) ..... A. indistintus (Barbosa \& Azevedo, 2011) gen. et comb. nov.

Astromesitius olavoi gen. et sp. nov.

urn:lsid:zoobank.org:act:0A21A488-42E3-4F07-B0F7-A4157ED1BC5B

Figs $1 \mathrm{~A}, 2 \mathrm{~A}, 3 \mathrm{~A}, 4 \mathrm{~A}, 5 \mathrm{~A}$

\section{Diagnosis}

Head longer than wide. Malar space shorter than VOL. First flagellomere as long as pedicel. Frontal angle of ocellar triangle a right angle. Mesosoma with dorsal pronotal area with anterior corner rounded, with side straight, posterior margin concave; longitudinal pronotal sulcus incomplete. Metapectalpropodeal complex as long as metapectal-propodeal complex half-width, with longitudinal ridge between metapostnotal median and metapostnotal-propodeal carinae; posterior projection of metapectalpropodeal complex present, short, thick, divergent. Lateral surface of metapectal-propodeal complex areolate, with upper carina and posterior carina. Mesopleuron lower half foveolate. Hypopygium as long as wide; anterior stalk wide and long; lateral projection long; posterior margin with branches wide. Genitalia with paramere apex sparse setose; ventral arm of paramere club-shaped, wide at apex; dorsal arm shorter than ventral arm, club-shaped, and with basal margin thin. Aedeagus aligned with paramere apex, apical margin truncate.

\section{Etymology}

The species is named in honour of Olavo Perim Galvão, a friend, an environmental auditor of the Instituto Brasileiro do Meio Ambiente e dos Recursos Naturais Renováveis (IBAMA) and a warrior for environmental causes, who died while fighting against deforestation in the Amazon, the place which he dearly loved. 

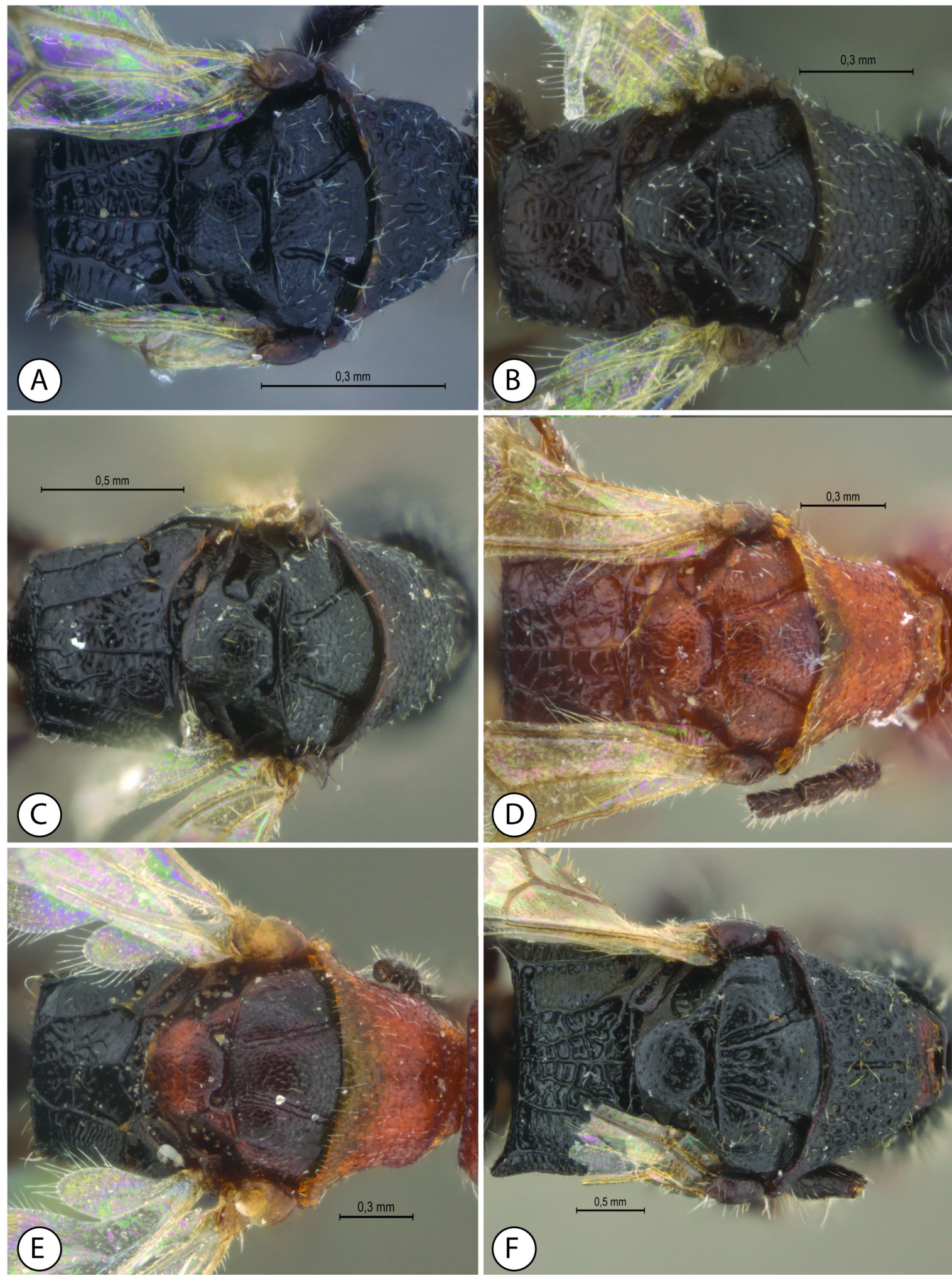

Fig. 1. Mesosoma of Astromesitius gen. nov., dorsal view. A. A. olavoi gen. et sp. nov. (holotype, QBG) B. A. thionyi gen. et sp. nov. (holotype, UFES 118956) C. A. carbonarius (Móczár, 1970) gen. et comb. nov. (UFES 56168) D. A. indistintus (Barbosa \& Azevedo, 2011) gen. et comb. nov. (paratype, UFES) E. A. minutissimus (Móczár, 1971) gen. et comb. nov. (holotype, UFES 56528) F. A. quatei (Móczár, 1977) gen. et comb. nov. (holotype, BPBM). 

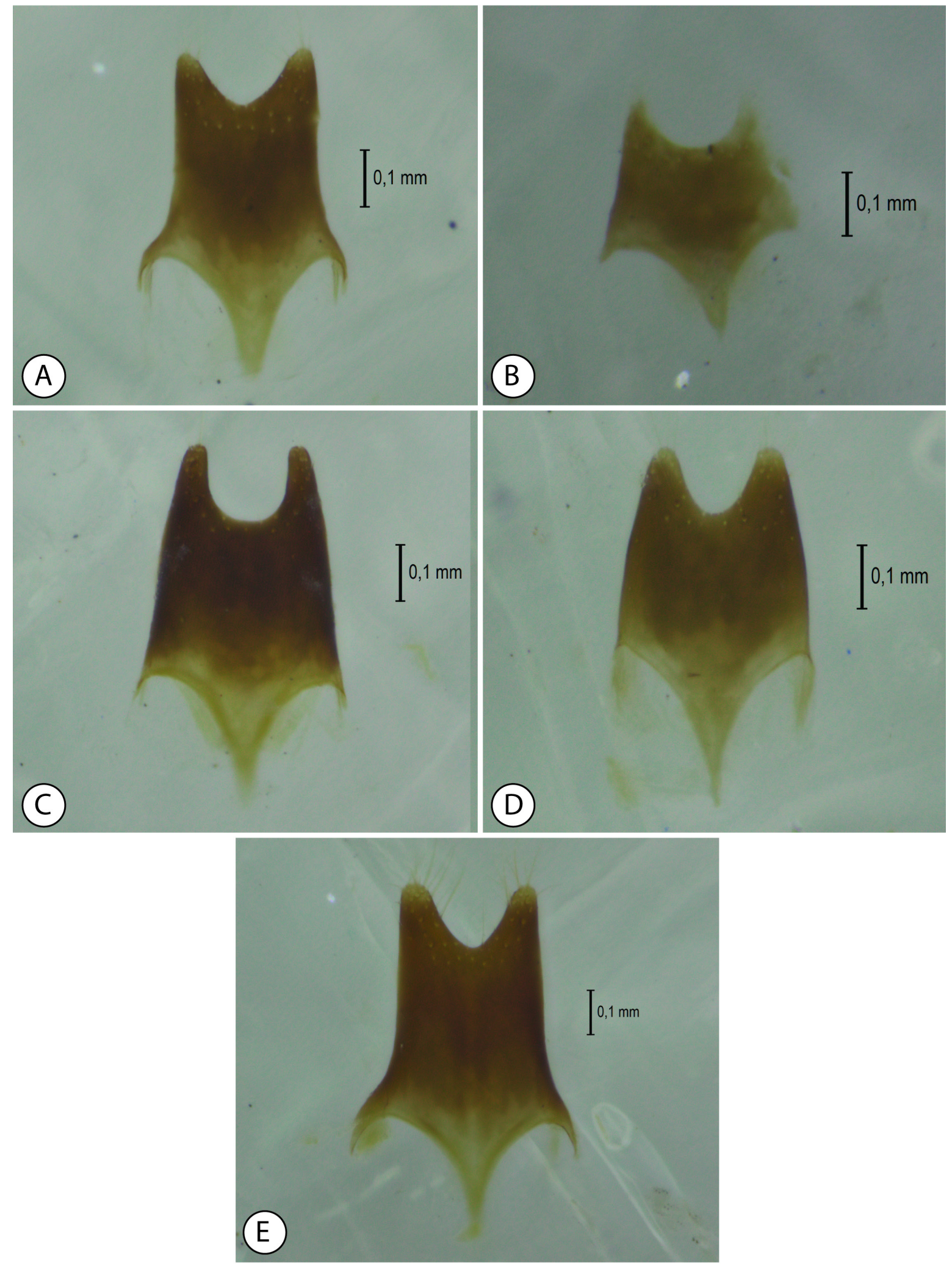

Fig. 2. Hypopygium of Astromesitius gen. nov. A. A. olavoi gen. et sp. nov. (holotype, QBG) B. A. thionyi gen. et sp. nov. (holotype, UFES 118956) C. A. indistintus (Barbosa \& Azevedo, 2011) gen. et comb. nov. (paratype, UFES) D. A. minutissimus (Móczár, 1971) gen. et comb. nov. (holotype, UFES 56528) E. A. quatei (Móczár, 1977) gen. et comb. nov. (holotype, BPBM). 


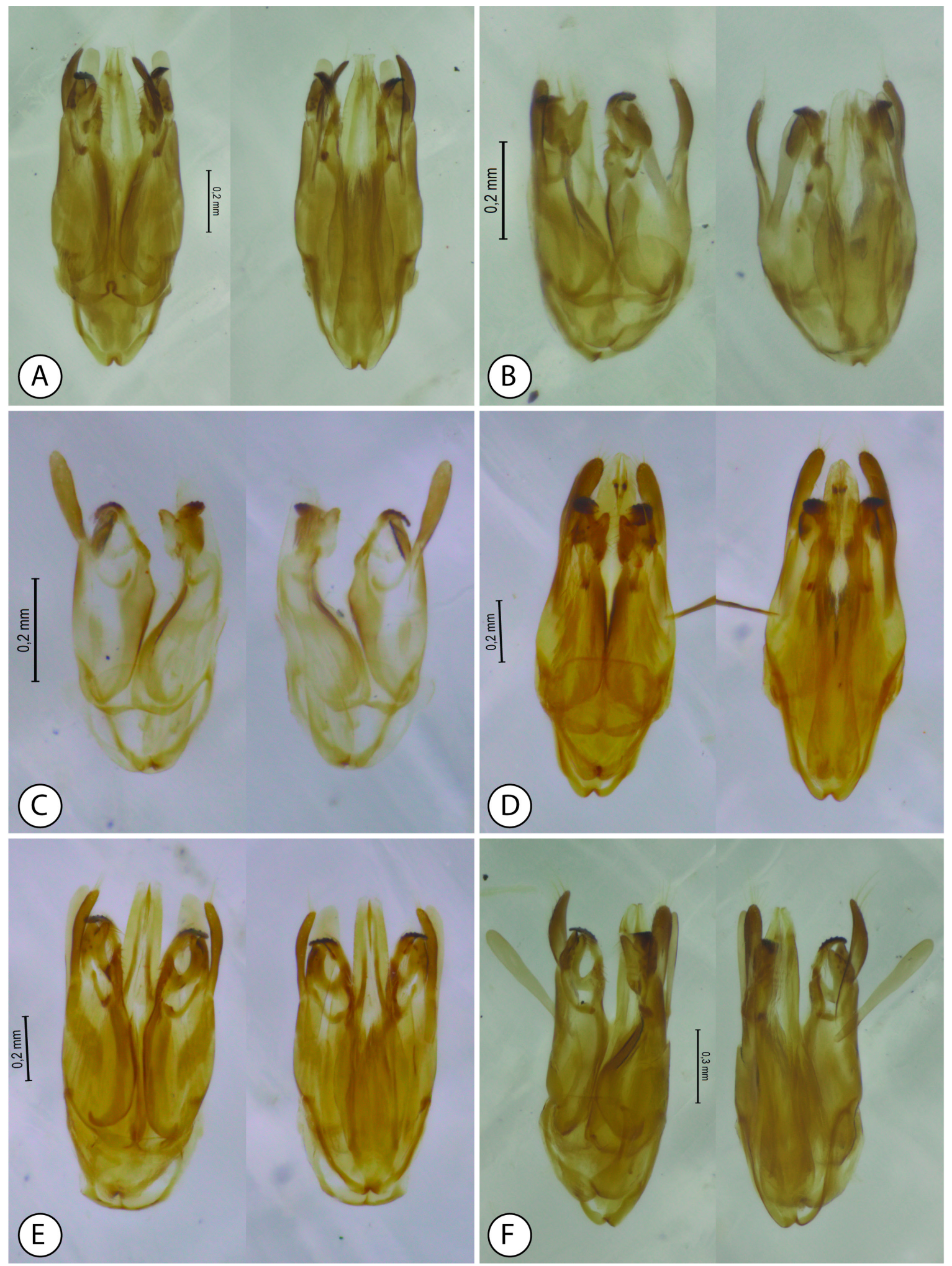

Fig. 3. Genitalia of Astromesitius gen. nov., left ventral and right dorsal view. A. A. olavoi gen. et sp. nov. (holotype, QBG) B. A. thionyi gen. et sp. nov. (holotype, UFES 118956) C. A. carbonarius (Móczár, 1970) gen. et comb. nov. (UFES 56168) D. A. indistintus (Barbosa \& Azevedo, 2011) gen. et comb. nov. (paratype, UFES) E. A. minutissimus (Móczár, 1971) gen. et comb. nov. (holotype, UFES 56528) F. A. quatei (Móczár, 1977) gen. et comb. nov. (holotype, BPBM). 

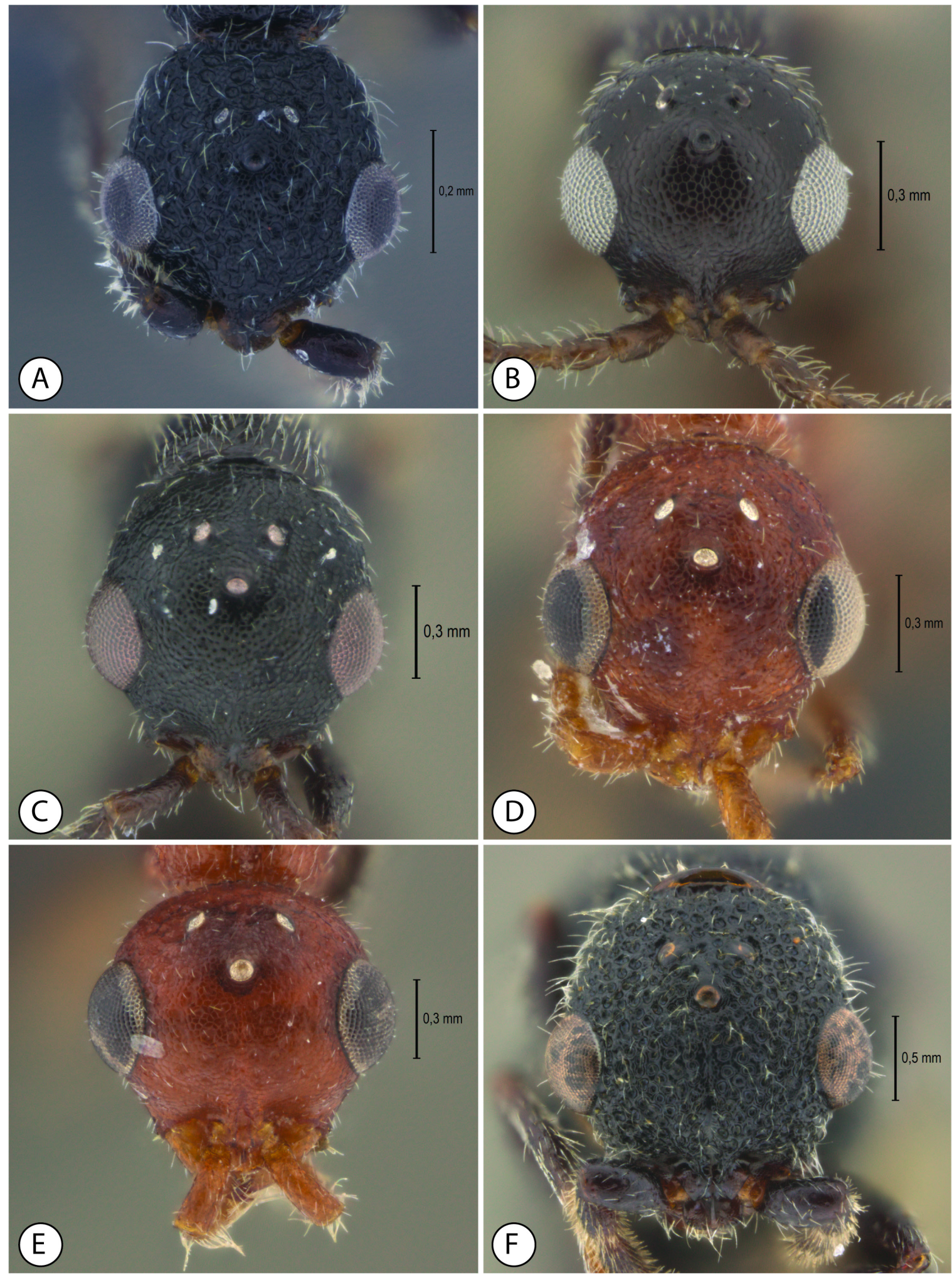

Fig. 4. Head of Astromesitius gen. nov., dorsal view. A. A. olavoi gen. et sp. nov. (holotype, QBG) B. A. thionyi gen. et sp. nov. (holotype, UFES 118956) C. A. carbonarius (Móczár, 1970) gen. et comb. nov. (UFES 56168) D. A. indistintus (Barbosa \& Azevedo, 2011) gen. et comb. nov. (paratype, UFES) E. A. minutissimus (Móczár, 1971) gen. et comb. nov. (holotype, UFES 56528) F. A. quatei (Móczár, 1977) gen. et comb. nov. (holotype, BPBM). 


\section{Material examined}

\section{Holotype}

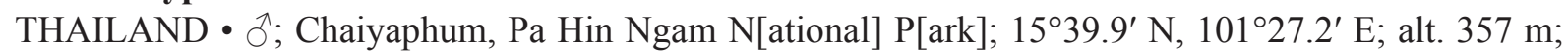
26 Dec. 2006-2 Jan. 2007; Katae Sa-nog \& Buakaw Adnafai leg.; deciduous; Malaise trap; QBG.

\section{Paratype}

THAILAND • 1 §ं; Ubon Ratchathani, Pha Taem N[ational] P[ark], Don Huay Can; $15^{\circ} 40.016^{\prime}$ N, $105^{\circ} 30.502^{\prime}$ E; alt. 246 m; 9-15 Jun. 2007; Tongcam \& Banlu leg.; Malaise trap; UFES.

\section{Description}

Body (Fig. 5A). 2.4-2.5 mm long; antenna 1.5-1.55 mm long; forewing $1.5 \mathrm{~mm}$ long.

Colour. Head and mesosoma black; clypeus and antenna dark castaneous almost black; mandible, palpi and legs dark castaneous, lighter distad; metasoma dark castaneous almost black; wings hyaline with venation light castaneous.

HEAD (Fig. 4A). Head longer than wide. Malar space shorter than VOL, parallel. Mandible with three sharp distal teeth. Median clypeal lobe quadrate, apical margin slightly outcurved, median clypeal carina complete, arched in lateral profile. Lateral clypeal lobe short. Antenna very long, reaching the middle of metasoma; scape slightly curved; pedicel caliciform; first flagellomere as long as pedicel; flagellomeres each almost twice as long as wide, proximal ones wider subdistally; flagellomeral pubescence erectsuberect, about $0.5 \times$ as long as flagellomeral diameter. HE about $0.35 \times \mathrm{LH}$. Frons strongly foveolate. Frontal angle of ocellar triangle a right angle. Occipital carina with surface foveolate.

Mesosoma (Fig. 1A). Dorsal pronotal area foveolate, shorter than wide, trapezoidal, anterior margin outcurved, anterior corner rounded, lateral margin concave, posterior margin concave; longitudinal pronotal sulcus incomplete, weakly impressed. Anteromesoscutum coriaceous, longer than mesoscutellum; longitudinal anteromesoscutal sulcus absent; notaulus deep, wide, polished, almost entirely straight, converging posterad; parapsidal signum shallow, narrow, straight, parallel. Mesoscutummesoscutellar sulcus straight medially, deep, and laterally dilated; mesoscutellum coriaceous. Metapectalpropodeal disc $0.5 \times$ as long as wide, with incomplete longitudinal ridge between metapostnotal median and metapostnotal-propodeal carinae; metapostnotal surface striate; metapostnotal median carina complete; posterior projection short, thick, discretely divergent posterad. Propodeal declivity areolate, with median and lateral carinae. Lateral surface of metapectal-propodeal complex areolate, subdorsal carina ill-defined, vertical carina complete. Mesopleuron foveolate, but progressively weakening posterad; posterior oblique sulcus foveolate; mesopleural suture with inner margin striate.

Wings. Fore wing with Rs\&M vein arched; pterostigma short, about as long as prestigmal abscissa of R1, distal margin convex. Hindwing with three distal hamuli, first hamulus widely separated from others.

Metasoma. Mostly polished, tergum I mostly glabrous, subsequent terga progressively more setose laterally and posteriorly. Hypopygium as long as wide; anterior stalk wide and long; lateral projection long; posterior margin with branches short and wide (Fig. 2A).

Genitalia (Fig. 3A). Paramere apex sparsely setose; ventral arm of paramere club-shaped, wide at apex; dorsal arm shorter than ventral arm, club-shaped, and with basal margin thin. Cuspis ventral arm not reaching dorsal arm apex, wide; dorsal arm wide. Aedeagus slender, with apex aligned with paramere apex, apical margin truncate, lateral margin of basal portion convex. Apodeme parallel, base curved laterad. 

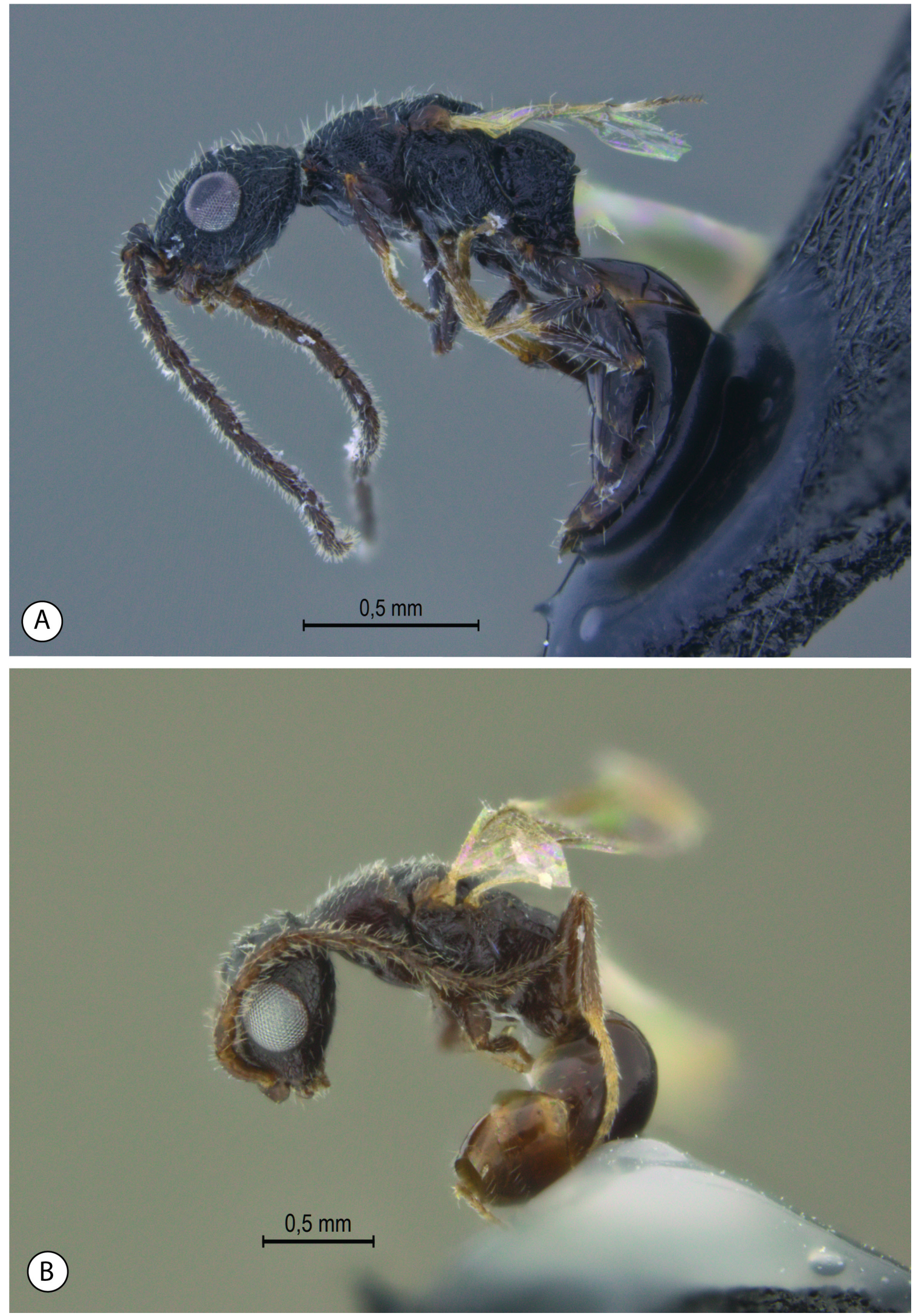

Fig. 5. Habitus of Astromesitius gen. nov. A. A. olavoi gen. et sp. nov. (holotype, QBG) B. A. thionyi gen. et sp. nov. (holotype, UFES 118956). 


\section{Remarks}

This species is similar to Astromesitius carbonarius (Móczár, 1970) and A. minutissimus (Móczár, 1971), mainly in having the small and black body; the head longer than wide; the hyaline forewing. Astromesitius olavoi gen. et sp. nov. can be distinguished from these two species by having the frons, the dorsal pronotal area and the mesopleuron sparsely foveolate; the dorsal pronotal area with longitudinal sulcus, but inconspicuous; the metapectal-propodeal disc with posterior projection inconspicuous; the genitalia with truncate and projected apex of aedeagus. Thus we did not doubt the validity of a new species.

\section{Distribution}

Thailand.

Astromesitius thionyi gen. et sp. nov. urn:1sid:zoobank.org:act:411E3776-71AD-4EFF-847E-334DFF06D0AC

Figs 1B, 2B, 3B, 4B, 5B

\section{Diagnosis}

Head as long as wide. Malar space shorter than VOL, parallel. First flagellomere as long as pedicel. Frontal angle of ocellar triangle acute. Mesosoma dorsal pronotal area with anterior margin convex, anterior corner rounded and side slightly concave; longitudinal pronotal sulcus incomplete. Metapectalpropodeal complex as long as metapectal-propodeal complex half-width, without longitudinal ridge between metapostnotal median and metapostnotal-propodeal carinae; metapostnotal surface polished; posterior projection of metapectal-propodeal complex absent. Propodeal declivity coriaceous. Lateral surface of metapectal-propodeal complex coriaceous, with upper carina and posterior carina. Mesopleuron coriaceous; mesopleural suture absent. Hypopygium as long as wide; anterior stalk wide and long; lateral projection short; posterior margin with branches slender. Genitalia with paramere apex glabrous; ventral arm of paramere sinuous, slender; dorsal arm as long as ventral arm, club-shaped. Aedeagal apex aligned with paramere apex, apical margin rounded.

\section{Etymology}

The species is named in honour of Thiony Emanuel Simon, a friend, post-doctoral fellow of the Graduate School of Zoology at UFES and marine researcher, who died while diving in a field expedition.

\section{Material examined}

\section{Holotype}

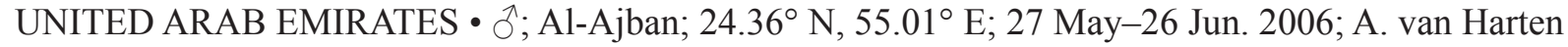
leg.; Malaise trap; UFES 118956.

\section{Paratypes}

UNITED ARAB EMIRATES $\bullet 1$; $7 \mathrm{~km} \mathrm{~S}$ of al-Jazirat al-Hamra; $25.40^{\circ} \mathrm{N}, 55.45^{\circ} \mathrm{E} ; 8$ Mar. 2005; A. van Harten leg.; hand collecting; UFES • 1 ○; Hatta x Huwaylat; $24.50^{\circ} \mathrm{N}, 56.09^{\circ} \mathrm{E}$; 12 Mar. 2005; A. van Harten leg.; hand collecting; UFES.

\section{Description}

Body (Fig. 5B). 1.3-1.8 mm long; antenna 0.9-1.1 mm long; forewing 0.8-1.0 mm long.

Colour. Head, clypeus, antenna, mandible, palpi, mesosoma, legs and metasoma dark castaneous, except tarsi castaneous. Wings hyaline, venation light castaneous. 
HeAd (Fig. 4B). Head as long as wide. Malar space shorter than VOL, parallel. Mandible with three sharp distal teeth. Median clypeal lobe quadrate, apical margin slightly outcurved, median clypeal carina complete, arched in lateral profile. Lateral clypeal lobe short. Antenna very long, reaching middle of metasoma; scape slightly curved; pedicel caliciform; first flagellomere as long as pedicel; flagellomeres each almost twice as long as wide; proximal ones wider subdistally; flagellomeral pubescence suberect, about $0.5 \times$ as long as flagellomeral diameter. HE about $0.42 \times$ LH. Frons coriaceous. Frontal angle of ocellar triangle acute. Occipital carina with surface foveolate; absent ventrally.

Mesosoma (Fig. 1B). Dorsal pronotal area coriaceous, shorter than wide, trapezoidal, anterior margin outcurved, anterior corner rounded, lateral margin concave, posterior margin concave; longitudinal pronotal sulcus very incomplete, restricted to anterior fifth, weakly impressed. Anteromesoscutum coriaceous, as long as mesoscutellum; longitudinal anteromesoscutal sulcus absent; notaulus deep, wide, polished, slightly incurved straight, converging posterad; parapsidal signum shallow, narrow, straight, parallel. Mesoscutum-mesoscutellar sulcus straight medially, deep, and laterally dilated; mesoscutellum coriaceous. Metapectal-propodeal disc $0.5 \times$ as long as wide, without longitudinal ridge between metapostnotal median and metapostnotal-propodeal carinae; metapostnotal surface striate; metapostnotal median carina complete, but weak and ill-defined posteriorly; posterior projection absent. Propodeal declivity very weakly areolate with polished background, median and lateral carinae very inconspicuous. Lateral surface of metapectal-propodeal complex very weakly areolate with polished background, subdorsal and vertical carina absent. Mesopleuron weakly coriaceous; posterior oblique sulcus weak, foveolate; mesopleural suture narrow.

WINGS. Forewing with Rs\&M vein arched; pterostigma short, about as long as prestigmal abscissa of R1, distal margin convex. Hindwing with three distal hamuli, first hamulus widely separated from others.

Metasoma. Mostly polished, tergum I mostly glabrous, subsequent terga progressively more setose laterally and posteriorly. Hypopygium as long as wide; anterior stalk wide and long; lateral projection short; posterior margin with branches short and slender (Fig. 2B).

Genitalia (Fig. 3B). Paramere apex glabrous; ventral arm of paramere sinuous, slender; dorsal arm as long as ventral arm, club-shaped. Cuspis ventral arm aligned with dorsal arm apex, slender; dorsal arm wide. Aedeagus slender, with apex aligned with paramere apex, apical margin rounded, lateral margin of basal portion slightly convex. Apodeme parallel, base straight.

\section{Remarks}

The species Astromesitius thionyi gen. et sp. nov. is slightly similar to A. indistintus, with which it shares the absence of a longitudinal ridge between the metapostnotal median carina and metapostnotalpropodeal suture. The new species is distinguished from all the other species of Astromesitius gen. nov. by the coriaceous body surface; the polished metapostnotal-propodeal complex surface; the metapostnotal median carina incomplete; the coriaceous propodeal declivity and lateral surface of the metapostnotalpropodeal complex; the genitalia with sinuous paramere ventral arm. Astromesitius thionyi gen. et sp. nov. is the smallest species of the genus and the characteristics cited above are exclusive to this species among Astromesitius gen. nov. species; thus we did not doubt the validity of a new species.

\section{Distribution}

United Arab Emirates. 
Astromesitius carbonarius (Móczár, 1970) gen. et comb. nov.

Figs $1 \mathrm{C}, 3 \mathrm{C}, 4 \mathrm{C}, 6 \mathrm{~A}$

\section{Diagnosis}

Body $2.1 \mathrm{~mm}$ long. LFW $1.5 \mathrm{~mm}$. Wings infuscate; head and mesosoma dark castaneous nearly black; metasoma dark castaneous. Head as long as wide. Malar space shorter than VOL, convergent anteriorly. Frons coriaceous. Ocelli small. Mesosoma with pronotal disc shorter than wide, coriaceous, with side straight, posterior margin sinuous; longitudinal pronotal sulcus absent. Metapectal-propodeal complex as long as metapectal-propodeal complex half-width, without longitudinal ridge between metaposnotal median carina and metaposnotal-propodeal suture; metaposnotal surface areolate; posterior spine of metapectal-propodeal complex absent. Propodeal declivity areolate. Lateral surface of metapectalpropodeal complex areolate. Metasoma with hypopygium as long as wide; lateral projection short; posterior margin with branches short and slender. Genitalia with paramere apex sparsely hairy; aedeagus slender, with apex not reaching paramere apex, apical margin angled, lateral margin of basal portion convex.

\section{Material examined}

UNITED ARAB EMIRATES • 1 đo; Al-Ajban; $24.36^{\circ} \mathrm{N}, 55.01^{\circ}$ E; 1 Apr.-2 May 2006; Malaise trap; A. van Harten leg.; UFES 56168.

\section{Remarks}

This species was originally described in Metrionotus (Móczár 1970a) from South Africa (Móczár 1970a), and was later recorded from the United Arab Emirates (Barbosa \& Azevedo 2011). We examined one male from UAE deposited at UFES, and the analyses reveal that their characteristics correspond to Astromesitius gen. nov. Thus, we propose a new combination for this species from Metrionotus to Astromesitius gen. nov.

Astromesitius indistintus (Barbosa \& Azevedo, 2011) gen. et comb. nov.

Figs 1D, 2C, 3D, 4D, 6B

\section{Diagnosis}

Body $2.2 \mathrm{~mm}$ long. LFW $1.2 \mathrm{~mm}$. Wings clear hyaline; head and mesosoma orange; metasoma dark castaneous. Head longer than wide. Malar space shorter than VOL, convergent anteriorly. Frons weakly foveolate. Ocelli small. Mesosoma with pronotal disc shorter than wide, weakly foveolate, with side straight, posterior margin concave; longitudinal pronotal sulcus absent. Antero-mesoscutum coriaceous. Metapectal-propodeal complex as long as metapectal-propodeal complex half-width, with longitudinal ridge between metaposnotal median carina and metaposnotal-propodeal suture; metaposnotal surface areolate; posterior spine of metapectal-propodeal complex present, short, thick, divergent. Propodeal declivity areolate. Lateral surface of metapectal-propodeal complex areolate. Metasoma with hypopygium longer than wide, anterior stalk wide and short; posterior margin with branches short and wide. Genitalia with paramere apex dense hairy; aedeagus slender, with apex aligned with paramere apex, apical margin rounded, lateral margin of basal portion slightly convex.

\section{Material examined}

Paratype

YEMEN • 1 §̊; Lahj; Aug. 2000; Malaise trap; A. van Harten \& A. Sallam leg.; UFES. 

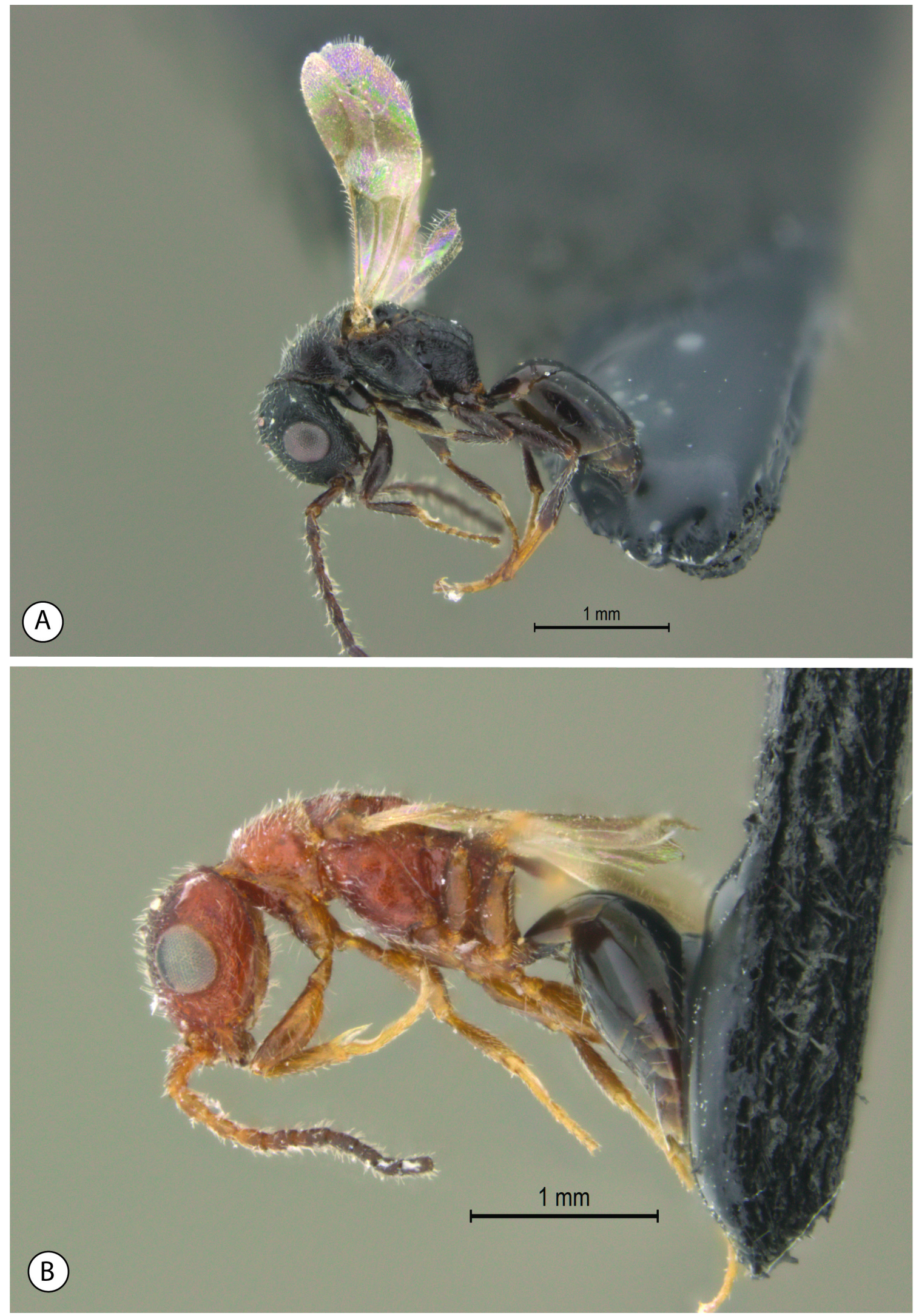

Fig. 6. Habitus of Astromesitius gen. nov. A. A. carbonarius (Móczár, 1970) gen. et comb. nov. (UFES 56168) B. A. indistintus (Barbosa \& Azevedo, 2011) gen. et comb. nov. (paratype, UFES). 


\section{Remarks}

This species was originally described in Metrionotus (Móczár 1970a) from the United Arab Emirates (Barbosa \& Azevedo 2011). We examined one male type, the paratype, from material deposited at UFES, and the analyses reveal that its characteristics perfectly fit Astromesitius gen. nov., including the star-shaped hypopygium. Thus, we propose a new combination for this species from Metrionotus to Astromesitius gen. nov.

Astromesitius minutissimus (Móczár, 1971) gen. et comb. nov.

Figs 1E, 2D, 3E, 4E, 7A

\section{Diagnosis}

Body $2.7 \mathrm{~mm}$ long. LFW $1.5 \mathrm{~mm}$. Wings infuscate; head and pronotum light castaneous; metasoma castaneous; mesosoma dark castaneous. Head as long as wide. Malar space as long as VOL, convergent anteriorly. Frons coriaceous. Ocelli large. Mesosoma with pronotal disc shorter than wide, weakly foveolate, with side straight, posterior margin concave; longitudinal pronotal sulcus hardly distinct, incomplete. Metapectal-propodeal complex as long as metapectal-propodeal complex half-width, without longitudinal ridge between metaposnotal median carina and metaposnotal-propodeal suture; metaposnotal surface areolate; posterior spine of metapectal-propodeal complex absent. Propodeal declivity striate. Lateral surface of metapectal-propodeal complex areolate. Metasoma with hypopygium longer than wide; anterior stalk wide and short; posterior margin with branches short and wide. Genitalia with paramere apex sparsely hairy; aedeagus slender, with apex surpassing paramere apex, apical margin rounded, lateral margin of basal portion slightly convex.

\section{Material examined}

Holotype

UNITED ARAB EMIRATES • ${ }^{\circledR}$; Wadi Bih dam; $25.48^{\circ} \mathrm{N}, 56.04^{\circ} \mathrm{E}$; $9-23$ Jul. 2008; light trap; van Harten leg.; UFES 56528.

\section{Remarks}

This species was originally described in Heterocoelia (Móczár 1971) from South Africa (Móczár 1971), and later recorded from the United Arab Emirates (Barbosa \& Azevedo 2011). It was transferred to Metrionotus by Móczár (1974). We examined the holotype male from UAE identified by Barbosa \& Azevedo (2011) and deposited at UFES, and the analyses, mainly of the hypopygium, reveal that their characteristics perfectly fit Astromesitius gen. nov. Thus, we propose a new combination for this species from Metrionotus to Astromesitius gen. nov.

Astromesitius mutilloides (Costa, 1864) gen. et comb. nov.

Clytrovorus mutilloides Costa, 1864: 126 (combined by Argaman, 2003: 75).

Mesitius fuscicornis Kieffer, 1906: 408, 535, 545 (synonymised by Argaman 2003: 75).

Mesitius zavadili Hoffer, 1936: 120-122, fig. 1 (synonymised by Argaman 2003: 75).

Mesitius viator Nagy, 1968: 171, fig. 4 (synonymised by Argaman 2003: 75).

\section{Diagnosis}

Female

Head as long as wide. Malar space shorter than VOL, convergent anteriorly. Eye very small. Frons foveolate. Ocelli very small. Mesosoma with pronotal disc as long as wide, weakly foveolate, with side straight, posterior margin concave; longitudinal pronotal sulcus hardly distinct, incomplete. Metapectalpropodeal complex shorter than metapectal-propodeal complex half-width, without longitudinal ridge 

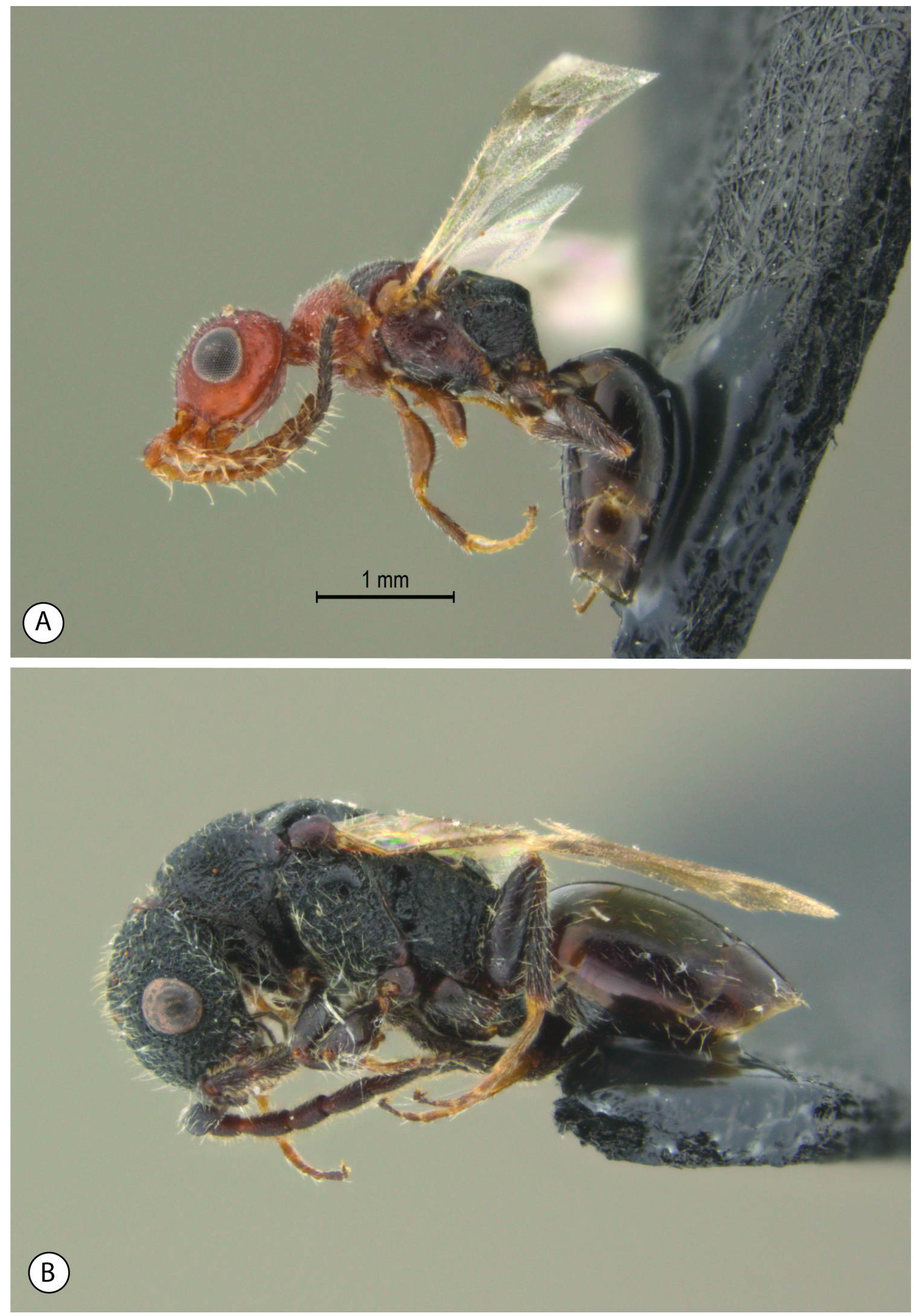

Fig. 7. Habitus of Astromesitius gen. nov. A. A. minutissimus (Móczár, 1971) gen. et comb. nov. (holotype, UFES 56528) B. A. quatei (Móczár, 1977) gen. et comb. nov. (holotype, BPBM). 
between metaposnotal median carina and metaposnotal-propodeal suture; metaposnotal surface areolate; posterior spine of metapectal-propodeal complex present, short, thin and divergent. Propodeal declivity striate. Wings micropterous.

\section{Male}

Hypopygium longer than wide; anterior stalk slender and long; posterior margin with branches short and wide. Genitalia with paramere apex sparsely hairy; ventral arm of paramere sinuous and slender; aedeagus slender, with apex aligned with paramere apex, apical margin rounded, and with short outer projection, lateral margin of basal portion slightly convex.

\section{Remarks}

This species was originally described in Sclerodermus (Costa 1864) based on one female from Italy. It was transferred to Mesitius by Kieffer (1914), who synonymised Sclerodermus mutilloides under Mesitius ghilianii Spinola, 1851. Argaman (2003) revalidated the status as a valid species and transferred this species from Mesitius to Clytrovorus. Argaman also established the synonymy of Mesitius fuscicornis Kieffer,1906, M. zavadili Hoffer, 1936 and M. viator Nagy, 1968 under this species.

We did not have access either to the type, which is lost, or to any other voucher specimens of this species, and the original descriptions are not sufficiently detailed to compare with the diagnostic characteristics of Astromesitius gen. nov. However, the illustration by Argaman (2003) is clear and sufficient to analyze and reallocate this species from Clytrovorus to Astromesitius gen. nov., because it has long and sparse antennal pubescence and the hypopygium is 'star-shaped'.

Astromesitius quatei (Móczár, 1977) gen. et comb. nov.

Figs 1F, 2E, 3F, 4F, 7B

\section{Diagnosis}

Body $4.3 \mathrm{~mm}$ long. LFW $2.5 \mathrm{~mm}$. Wings bi-banded; head and mesosoma dark castaneous nearly black; metasoma dark castaneous. Head longer than wide. Malar space shorter than VOL, convergent anteriorly. Eye very small. Frons foveolate. Ocelli large. Mesosoma with pronotal disc as long as wide, foveolate, with side slightly concave, posterior margin concave; longitudinal pronotal sulcus hardly distinct, incomplete. Metapectal-propodeal complex shorter than metapectal-propodeal complex halfwidth, with longitudinal ridge between metaposnotal median carina and metaposnotal-propodeal suture; metaposnotal surface areolate; posterior spine of metapectal-propodeal complex present, short, thick, parallel. Propodeal declivity areolate. Lateral surface of metapectal-propodeal complex areolate. Metasoma with hypopygium longer than wide; anterior stalk slender and short; posterior margin with branches short and wide. Genitalia with paramere apex glabrous; aedeagus slender, with apex aligned with paramere apex, apical margin rounded, lateral margin of basal portion slightly convex.

\section{Material examined}

Holotype

VIETNAM• O’; Djiring; alt. 1200 m; 22-28 Apr. [19]60; Grasse, L.W. Quate leg.; BPBM.

\section{Remarks}

This species was originally described in Sulcomesitius and recorded from Vietnam (Móczár 1977). We examined the holotype male deposited at BPBM, and the analyses reveal that it fits the concept of Astromesitius gen. nov., mainly because of the hypopygium shape. Thus, we propose a new combination for this species from Sulcomesitius to Astromesitius gen. nov. 


\section{Discussion}

Since the Mesitiinae synopsis by Argaman (2003), the hypopygium has played a very important taxonomic role at a genus level within the subfamily. Argaman postulated that each genus has a conservative hypopygium morphology, with very little variation within each one, but with huge morphological distinctions among the genera.

Five species of Metrionotus, Clytrovorus and Sulcomesitius (see 'Included species' above) were recognised as possessing a 'star-shaped' hypopygium. The 'star-shaped' hypopygium has as its main characteristic the latero-anterior arm developed, and this development could be associated with the muscle insertion. Schulmeister (2003) recognised the lateral S9-cupulal muscle; this muscle originates from the gonocondyle in the cupula of the genitalia and inserts laterally on the ninth sternite (hypopygium). The cupula is attached to the base of male genitalia, and the muscles between the cupula and the hypopygium enable some movements of genitalia structures. Thus, the lateral S9-cupulal muscle exerts direct action on the male genitalia. According to Boudinot (2013) this muscle torques the genitalia by the elevation of the cupula basal margin; probably the greater insertion point could increase the torque movement. Thus, the long latero-anterior arms of the 'star-shaped' hypopygium might give an additional functional advantage during copulation in comparison to the other genera of Mesitiinae. It could ensure a better muscle insertion and improve the muscle action of male genitalia movement. Moreover, this distinct shape of hypopygium makes Astromesitius gen. nov. easy to recognise among the other genera of Mesitiinae.

There are some other important characteristics to diagnose the genus. One of them is the surface sculpture of the integument. Most Mesitiinae have the integument surface densely impressed with large foveae and with a longitudinal median sulcus on the mesosoma, characteristics that could be an evolutionary novelty for this subfamily. Astromesitius gen. nov., however, has the body sparsely foveolate or almost not foveolate and the longitudinal median sulcus hardly distinct or absent. The genera Anaylax Móczár, 1970, Bradepyris Kieffer, 1905, Clytrovorus and Moczariella Barbosa \& Azevedo, 2014 - see Móczár (1970b), Barbosa \& Azevedo (2014), Nagy (1972) and Barbosa \& Azevedo (2014), respectively - have the integument with weak sculpture and without a longitudinal sulcus crossing the dorsal mesosomal area, or when present it is hardly distinct. In Mesitiinae, the more foveolate integument is related to a more conspicuous longitudinal sulcus of the mesosoma and a larger posterior projection of the metapectal-propodeal complex (see details in Azevedo et al. 2018).

The antennal pubescence in Mesitiinae can be short, medium or long, thick or thin, and dense or sparse (Argaman 2003; Móczár 1970a, b). However, in most genera the antennal pubescence is short, only in Incertosulcus Móczár, 1970 and Astromesitius gen. nov. the antennal pubescence is medium or long. Thus, although this condition is not exclusive to Astromesitius gen. nov., it helps conceptualise the genus. As emphasised by Riolo et al. (2016), antennal pubescence and sensilla play an important role in perception, therewith, this pubescence length could give additional adaptation fitness to Astromesitius gen. nov. species, thus becoming diagnostic for this genus.

\section{Acknowledgements}

We thank Antonius van Harten, Michael Sharkey and James Boome for providing the material studied. We thank Denis Brothers for elucidating doubts about the nomenclatural acts. We also acknowledge grants from Conselho Nacional de Desenvolvimento Científico e Tecnológico (CNPq), \#473386/2008-9 and \#620064/2006-4, the Taxonomy program, \#563953/05-5 and \#562224/2010-6, Fundação de Amparo à Pesquisa do Espírito Santo (FAPES), \#3935842/2007 and \#41106407/2008, for the bursary granted to the first author and CNPq grant \#303748/2018-4 for the bursary for the second author. We thank two anonymous reviewers for the useful suggestions. 


\section{References}

Argaman Q. 2003. Generic synopsis of Mesitinae Kieffer, 1914 (Hymenoptera: Bethylidae). Entomofauna 24: 61-96.

Azevedo C.O., Alencar I.D.C.C.A., Ramos M.S., Barbosa D.N., Colombo W.D., Vargas J.M. \& Lim J. 2018. Global guide of the flat wasps (Hymenoptera, Bethylidae). Zootaxa 4489: 1-294. https://doi.org/10.11646/zootaxa.4489.1.1

Barbosa D.N. \& Azevedo C.O. 2011 Order Hymenoptera, family Bethylidae, subfamily Mesitiinae. In: Harten A. van (ed.) Arthropod Fauna of UAE. Vol. 4: 375-404. Dar Al Ummah Printing, Publishing, Distribution \& Advertising, Abu Dhabi.

Barbosa D.N. \& Azevedo C.O. 2014. Moczariella, a bizarre new genus of Mesitiinae (Hymenoptera, Bethylidae) from Arab Peninsula. Zootaxa 3860: 291-297. https://doi.org/10.11646/zootaxa.3860.3.7

Boudinot B.E. 2013. The male genitalia of ants: musculature, homology, and functional morphology (Hymenoptera, Aculeata, Formicidae). Journal of Hymenoptera Research 30: 29-49.

https://doi.org/10.3897/jhr.30.3535

Costa A.C.H. 1864. Anno II - 1862 con quattro tavole. Parte prima et seconda. Annuario del Museo Zoologico della R. Università di Napoli 2: 1- 176.

Dallwitz M.J., Paine T.A. \& Zurcher E.J. 1993. User's guide to the DELTA system: a general system for processing taxonomic descriptions, Fourth Ed.

Available from https://www.delta-intkey.com/www/uguide.htm [accessed 20 Nov. 2019].

Harris R.A. 1979. A glossary of surface sculpturing. Occasional Papers in Entomology 28: 1-31.

Hoffer A. 1936. Rod Mesitius Thoms. v Československé republice. Genus Mesitius Thoms. in der Čechoslovakei. (Bethylidae, Hym. - Vespoid.). Acta Societatis entomologicae cechosloveniae 33: 119124.

Kawada R. \& Buffington M.L. 2016. A scalable and modular dome illumination system for scientific microphotography on a budget. PLoS ONE 11 (5): e0153426.

https://doi.org/10.1371/journal.pone.0153426

Kieffer J.-J. 1906. Proctotrypides. In: André E. (ed.) Species des hyménoptères d'Europe \& d'Algérie. Vol. 9: 289-551. A. Hermann, Paris.

Kieffer J.-J. 1914. Bethylidae. Das Tierreich 41: 228-595.

Móczár L. 1970a. Mesitiinae of world, genera Sulcomesitius Móczár and Metronotus Móczár. II. (Hymenoptera: Bethylidae). Acta Zoologica Academiae Scientiarum Hungaricae 16: 409-451.

Móczár L. 1970b. Mesitiinae of world with new genera and species. I. (Hymenoptera: Bethylidae). Acta Zoologica Academiae Scientiarum Hungaricae 16: 175-203.

Móczár L. 1971. Mestinae of world, genera "Mesitius spinola", Pilomesitius Móczár, Parvoculus Móczár, Pycnomesitius Móczár and Heterocoelia Dahlbom. III. (Hymenoptera: Bethylidae). Acta Zoologica Academiae Scientiarum Hungaricae 17: 295-332.

Móczár L. 1974. On another species of the genus Metrionotus Móczár (Hymenoptera: Bethylidae: Mesitiinae). Acta Biologica, Szeged 20: 173-177.

Móczár L. 1977. A review of the genus Sulcomesitius Móczár (Hymenoptera: Bethylidae, Mesitiinae). Acta Zoologica Academiae Scientiarium Hungaricae 23: 139-170.

Nagy C. G. 1968. A new record of Mesitinae (Hymenoptera, Bethylidae). Memorie della Società Entomologica Italiana 47: 168-176. 
Nagy C.G. 1972. Taxonomic remarks on Mesitinae (Hymenoptera Bethylidae). Memorie Societa Entomologica Italiana 51: 5-18.

Riolo P., Isidoro N., Ruschioni S., Minuz R.L., Bin F. \& Romani R. 2016. Anatomy of the antennal dorsal organ in female of Neodryinus typhlocybae (Hymenoptera: Dryinidae): a peculiar sensory structure possibly involved in perception of host vibration. Journal of Morphology 277: 128-137. https://doi.org/10.1002/jmor.20485

Schulmeister S. 2003. Genitalia and terminal abdominal segments of male basal Hymenoptera (Insecta): morphology and evolution. Organisms, Diversity and Evolution 3: 253-259.

Manuscript received: 2 July 2019

Manuscript accepted: 18 September 2019

Published on: 11 December 2019

Topic editor: Gavin Broad

Desk editor: Radka Rosenbaumová

Printed versions of all papers are also deposited in the libraries of the institutes that are members of the EJT consortium: Muséum national d'histoire naturelle, Paris, France; Meise Botanic Garden, Belgium; Royal Museum for Central Africa, Tervuren, Belgium; Royal Belgian Institute of Natural Sciences, Brussels, Belgium; Natural History Museum of Denmark, Copenhagen, Denmark; Naturalis Biodiversity Center, Leiden, the Netherlands; Museo Nacional de Ciencias Naturales-CSIC, Madrid, Spain; Real Jardín Botánico de Madrid CSIC, Spain; Zoological Research Museum Alexander Koenig, Bonn, Germany; National Museum, Prague, Czech Republic. 